Accretion 193, 198

Accretion disks (see also "disks") 9, 11ff, 23ff, 52, $97,107 \mathrm{ff}, 117 \mathrm{ff}, 179,183-$ 4, $316 \mathrm{ff}$

Accretion region (see also "HTAR" ) 73

Alfvén radius 169,319

Algol, photometry of 37-8

Algols, reverse $279 \mathrm{ff}$

$\alpha$ disks 25, 109

Angular-momentum loss 141-2, $165,212,318,348$

Angular-momentum transfer (transport) 232,314

Balmer emission (see also " $\mathrm{H} \alpha$ emission") 9, 22

Barium stars $168,170,172-3$, 177

Barnes-Evans calibration 67

B Lyrae, light-curve 287, variability of light-curve $39 \mathrm{ff}, 91$

B Lyrae paradox 216

Binaries in clusters 333,346

Braking, magnetic 141 passim, $171-2,188,255,289$

tidal 193

Bremsstrahlung, magnetic 226

Ca II emission 223ff, 230, 232

Cataclysmic binaries (variables) $7,90,117,125,161,167$, $172,299,318$

Catalogues (of Algols) 205, $217,327-8$

Chandrasekhar effect 63,77

Chandrasekhar limit (mass) $158,173,305-6$

Chromospheric activity (in binaries) 219ff, 226-7, 230,232

Circumstellar emission (radiation) $96,312,317$

Circumstellar material 4, 10 , $95,192,317-8$
CNO abundances $6,89,139,151,248$, $252 \mathrm{ff}$

Coalescence 174

Colour change (transient in Algo1) 372

Common-envelope evolution (phase)

128,171 passim, 299ff, 309, 331

Contact phase $127,129,289$

CORAVEL $180 \mathrm{ff}, 187$

Corona 225,311

Cross-correlation (radial velocities) $180,181 \mathrm{ff}, 187$

Cycles, magnetic $229 \mathrm{ff}, 312$

\section{DAOPHOT 218}

De Laval nozzle 317

Disks (see also "accretion disks") $16,19-20,107 \mathrm{ff}, 117 \mathrm{ff}, 319$, $324,331 \mathrm{f}, 340$

formation of 109-10

in $\varepsilon$ Aur 336

Keplerian 52, 110

modelling of 118

optically thin 119

permanent 59,112

"sub-Keplerian" 61, 111-2, 317

transient $53,57,112,317$

velocity structure of $55 \mathrm{ff}$

Distribution of Algols

by period 208

by primary mass 208-9

by spectral type 207-8

in space $6,7,146-7$

on the sky 206-7

Doppler broadening 241

Double contact $195,198,236,238$

Dynamos 314-5

Dust in circumstellar matter 25, $323,332,356,362$

Eddington approximation 121-2

Evolution

by mass transfer 128

of Algols 127,158

of low-mass Algols 148

to contact states 289 
Fate of low-mass Algols 149

Fe II, Fe III emission 101

Fields, magnetic $228,311 \mathrm{ff}$

FK comae stars 220

Formation of Algols 127, 143, $155-6,369$

Galaxy 5, 147, 189, 329

Gravity darkening 353

GaAs tubes 257-8, 268

$\mathrm{H} \alpha$ emission $10,11,14,358$

Hertzsprung gap 150,158 , 163,378

Hertzsprung-Russel1 diagram $339,357,378$

HRVR (High-radial-velocity region) 328-9, 358

HTAR (High-temperature accretionregion) $55,60,73,318$

Hubble time 150,306

Hydrogen content 131

Impact (see also "splash")

97

IR excess 226

Irradiation (of Algol secondaries) 272,343

Kepler's laws 345

Kurucz atmospheres 105, 272, 343,371

Lagrangian point, inner $\left(\mathrm{L}_{1}\right)$ $97,221,293,316-7,332$

Layers, high-temperature $122 \mathrm{ff}$

Leibnitz theorem 270, 278

LIGHT 288,326

Light-curve synthesis 326

Light-synthesis optimization $269 \mathrm{ff}$

Magnetic activity (see also

"fields") $72,143,318$

Mass flow 82, 299

Mass loss 36

Mass-ratio (critical) 160

(initial) 162

Mass transfer (exchange) 36, 66, $69,72,108,131,146,155$, $160,179,192-3,200,220$, $227,300,313 \mathrm{ff}, 338,350$
Case A 66, 130ff, 165, 200, $289-90,361$

Case B $66,135-6,165-6,200,369$

Case C 165

pulsed $174,176,210$

reverse 139,339

Mixing, thermohaline 134

Multiple subsets, method of 273 , 278

Na D lines 247

Nebulae, planetary $171,303-4,309$

Oscillations, rapid 352

Paradox ( $\beta$ Lyr) 216, (2nd Algol) 166

Particle trajectories $109 \mathrm{ff}$

Period changes $27,40,85,179,183$, $188-9,227-8,313,316,325,330$

Period, critical 200

Planck function 121 opacity 122-3

Plasma radiation 97,105

Polarization,

circular 72,77

in Algols $63 \mathrm{ff}$

interstellar $66 \mathrm{f}$

intrinsic $66 \mathrm{ff}$

radio 68

variable (sporadic) 68, (with

phase) 72,189

Polytropes, condensed 160, 164

Radio emission 226, 232-3, 367, 370

Ram pressure 115

Rayleigh scattering 25

Relaxation (of mass-gaining star) 337

Reverse algols $279 \mathrm{ff}$

Rings, gaseous 9, 51

Roche lobe (surface, equipotential) $4,16,18,24,26,37,49,80-1$, $139,143,145,153,157$ passim, $168 \mathrm{ff}, 180,186,201,205,215$ passim, 233, 236, 238, 269, 273, $280,288 \mathrm{ff}, 299,305,307,323-4$, 330,335 passim, 350,369

Roche-lobe overflow (RLOF) 52, 83, 133,142 passim, $165 \mathrm{ff}, 171$ passim, 187-8, 200, 209-10, 252, 
$325,330-1$

Rosseland mean opacity 122

Rossiter effect 237

Rotation 193, 236

critical $89,184,201,203$, 238

synchronous $185,201,236$, 238

rates (velocities) 183, 222, $235,237,240 \mathrm{ff}, 368,373$

Rotational lobe 236

Rotational statistics $196-7$

RS CVn stars 2, 143, 167-8, $192,219 \mathrm{ff}, 224 \mathrm{ff}, 230 \mathrm{ff}$, $295,312,324-5,330,351$, $357,362,369-70$

Russell-Merrill method 184 , 326-7

S giants 173

Scattering, by dust 26

Scattering envelope 69

Selection, observational 3, 217

Serpentids - see W ser stars

Shell spectrum (SX and RX Cas) 184

Signal-to-noise ratio 245,253

Skumanich's law $142,151,153$, 319

Spectroscopy, time resolved 523

Spin-orbit coupling 227

Spiral-in $174,302,309$

Splash 99, 110, 115, 328, 331

Spots 2, 224ff, 230, 233, 315

Streams, gaseous $4,11,22,112$, 316,375

Subgiant, undersize 152

Sun $38-9,313,320,322$

Supernova $173,299,305,307$

Symbiotic stars $167-8,170$

Technetium 173

T Tauri stars 117,220

Turbulence 125, (supersonic) 318

UPS (Utrecht Photometric System $257 \mathrm{ff}, 263-4,266,371$

UV astronomy 80 excess 226 spectrometry 85

spectroscopy 95

Velocity, terminal wind 70-1, 319

W Serpentis, model of 46-7

W Serpentis stars $35,99,104,179$ passim, 188, 191ff, $198 \mathrm{ff}, 324$, $333,363-4$

White dwarf

companions $172-3,219$

double $168,173,299,305 \mathrm{ff}$

helium 146,149

Wilson-Devinney Code (method, etc.)

$184,238,259,262,264,269-70$,

$279-80,282,288,326,357,359$, 371,376

Wind,

conical 125,326

stellar $66,100,146,165,172$,

$176,188,289$

magnetic stellar $100,148-9,318-9$

WINK $326,374,376$

Wolf-Rayet binaries 200, 209, 350

W UMa light-curve 287

W UMa stars (contact binaries) 145, $192,220,287,292$ passim, 318, $329,348,354$

X-ray binaries 299

$x$-ray emission $225,230,232$

Zeeman broadening 233 